\title{
Perforación gástrica en el recién nacido
}

\author{
Dr. Pablo Valenzueld H. 1 ; Dr. Gonzalo l!rcelay M. ${ }^{1}$
}

\section{Gastric perforation in a newborn}

Gastric perforation ocurred in the second day of life in an apparently healthy newbom of 36 weeks gestational age and was succesfully repaited, but recurred eight days later. At the second surgical intervention a previously inadveted intestinal makotation was detected and corrected obtainum normal intestinal transit. At 60 days of life he was submited to surgical intervention for intestinal obstruction caused by peritoneal adhesions without evidence of perforation. Thi patient died later from weondary peritonitis, that was confirmed at authopsy. Castrointestinal perforations were not found in this later examination.

(Key words: newborn, gastric perforation, intestinal naaltotation,)

La perforación gástrica del reciến nacido ( $P G)$ es una emergencia quirúrgica rara en nuestro medio, sin embargo debe tenerse presente ya que la subrevida está en directa relación con el diagnóstico preco $z$ y resolución quirúrgica oportuna.

En la literatura nacional sólo se ha publicado un caso de perforación gástrica espontánea $(\mathrm{PGE})^{\mathbf{1}}$. A propósito de un episodio característico ocurrido en la matemidad del Fospital C: $\mathrm{l}_{1}$. nico de la Unjersidad de Chile nos ha parecido de interés su análisis clínico y revisión de la bibliografía.

\section{CASO CLINICO}

Varón nacido de smbarizo controlado de exolución normal, parto espontánco eutóicico, 36 semanas de edad gestacional, pesó $2.800 \mathrm{~g}$. Apgar 9 y 9,1 y 5 minutos. Alimentado al pecho desde las 6 horas de vida, in problemas hasta el scgundo día en que pot bilirrubjnemia total de 13,5 mg fuc colocudo en fototerapid. Dos y medio días después apareció distensión abdominal y vómitos biliosos. La radiogtafía de abdomev reveló neumopetitoneo, colapso visceral $y$ nivel hidroaerco (figura 1). Fue trasiadado al Hospital Roberto del Río para su tratamiento quírúrgico; al ingsesar se veía grave, sin síguos de shock; abdomen distendido, blando y doloroso, ruidos intestinales ausentes, matide 2 hepáticu abolida y halo eritematoso periumbilical con ombligo lımpio. tn la intervención quirúrgica se encontró gran cantidad de aire en cavidad abdominal y líquido peritoneal claro. Fn cara anterior del estómago, contirun al borde de la curvatura mayor, habja una perrorición de 1

I. Lnidad de recién nacidos del Hospital Clínico do la Universidad de Chile y Hospital Roberto del Rio por $2 \mathrm{~cm}$, en una zona desprovista de músculo y serosa Se reparó la pared con sutura en tres planos y se dejó sonda nasogástrica. El curso postoperatorio fue normal. Sc indicó alimentación parenteral desde el primer día y

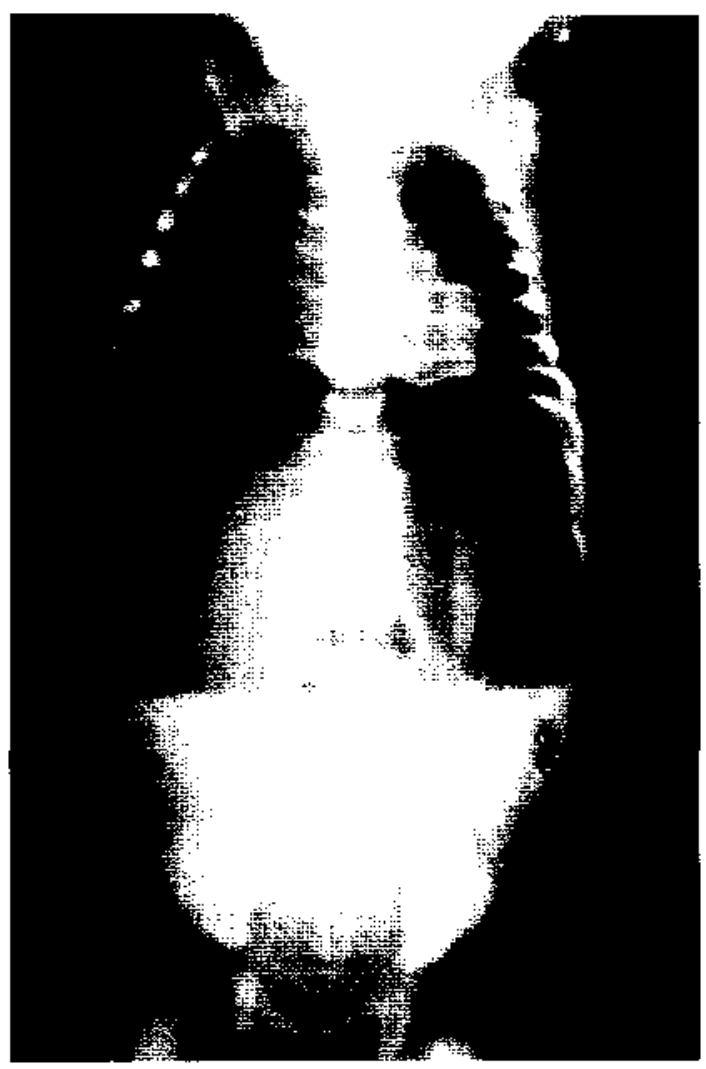

Figura 1: Colapso visceral, neumoperitoneo y nivel lijdroáreo intraperitoneal en la perforación gástrica de recién natido. 
oral desde el $5^{\circ}$ con leche materna. Como presentaba residuo gástrico importante se efectuó un estudio radiológico que reveló una obstrucción duodenal, reoperándose 8 días después de la primera operación. Lista vez hahía una pertoración gástrici en la cará anterior del estómago, bridas duodenales, perforación cubicrta dol jteon terminal y malrotación istestinal. Se suturaron las perfotaciones, debridó y fijó el çego a la fosa jlíaca derecha (FID). Se réalizó gastrostomía y drenaje parietocúlico derecho. Postopcratorio sin problemas. Profilaxis con cefotaxinta y amikacina por 10 días. Siete días despuess el tránsito intestinal cra normal, rejniciándosc alimentación enteral al día siguiente; para darse su alta a los 37 dias de nacido, pesando $2.710 \mathrm{~g}$.

Reingresó 20 días despućs por vómitos biliosos, distensión abdominal $y$ constipación, cn malas cundiciones generaics. La radiografía teveló niviles hidroaéreos operándose de urgencia. En esta tercera operación sc oncontraron y liberaron adherencias abundartes. Sc fjjó el intestino delgado. Sufrió shock séptico en el postoperatorio, lalleciendo a las $36 \mathrm{~h}$ de operado. Los hemocultivos premortem desarollaron E. coli. En la necropsia habia evidencia de peritonitis aguda. Yo habian perforaciones en las asas intestinales y el estómago tenía cicatrices de perforaciones antiguas.

\section{COMENTARIO}

Se habla de perforaciones espontáneas para hacer referencia a aquellas que ocurren en intes. tino aparentemente normal y sin causa obvia, a diferencia de las secundarias que en genctal se. rían determinadas por obstrucciones intestinales. Aparentemente en ambas habría una lesión ana. tómica o histológica en las paredes del tubo dj. gestivo.

Las primeras publicaciones sobre este problema aparecieron en 1925 y $1928^{2}$, oportunidad en que se intentó por primerá vez el tratamiento quirúrgico, asumiéndose que el cuadro equivalía a una úkera gastrica del adulto. En 1943 se informó sobre una deficiencia congénita de la pared gástrica y sólo en 1950 se logró la primera operación exitosa, atribuyéndose la causa de la perforación a una deficiencia de la pared gástri$\mathrm{ca}^{3}$. De 44 casos de $\mathrm{PG}, 22$ correspondían a perforaciones de la curvatura mayor y 4 cerca de la menor. En $1965^{4}$ se puso en uluda la falla exclusiva de la pared gástrica y se postuló como principal causa la sobredistensión gástrica.

fzzo y cols. ${ }^{1}$ presentaron cuatro casos de perforación gastrointestinal espontánca operados en el hospital Luis Calvo Mackenna, uno de los cuales se ubicaba en el eștómago. En una revisión de la casuística entre los años 1951 y 1964 se registraron 11 casos (incluyendo los de la publicación anterior). 8 espontáneos y 3 secundarios a una obstruccion ${ }^{10}$. Desde entonces no hay comunicaciones al respecto en la literatura médica nacional.

Dentro de las causas de perforación gastroin. testinal $1^{5-9,11}$ se debe descartar primero toda afección que lleve a la obstrucción mecánica o funcional responsables de perforaciones secundarias (atresias, ileo meconial, vólvulo, megacolon, etc.). Si éstas no se encuentran $y$ se pueden descartar causas iatrogénicas o traumáticas ( $\mathrm{SNG}$, tubos, enemas), se podrá plantear que la perforación es espontánea o jdiopática.

Entre los factores que podrían contribuir a la perforación, la hipoxia seria determinante en la lesión de la mucosa y pared, pues la anatomía patológica muestra generalmente lesiones isquémicas alrededor de la perforación. La hipoxia tisular estaría precedida por asfixia o por is. quemia prolongada, que provocarían fenómenos similares a la enterocolitis necrotizante. Sin embargo, no siempre se asocia a la asfixia y no se han informado casos de $P G$ asociado a la enterocolitis necrotizante. Además, estudios experimentales demuestran que la sobredistensión gástrica produce rotura del estónago a nivel de la curvatura mayor. Las causas de dilatación gás. trica neonatal son numerosas (obstrucciones bajas, uso de respiradores con presión negativa externa o presión positiva intermitente, de la vía aérea, hernja diafragmática). Varias razones teó ricas sugieren la existencia de un punto de menor resistencia en la curvatura mayor del estómago: en esa zona, hacia el cardias, la pared es más delgada, y según la ley de Laplace, que establece una relación directa entre la presión, tensión y radio, en la curvatura mayor y el fondo gástrico la tensión sería mayor. Por otra parte, existen en la pared del estómago del recién nacido zonas con ausencia de trama de la muscular, especialmente en el fondo gástrico y la curyatura mayor, más comunes en recién nacidos prematuros. De este modo, un feto con el estómago lleno de líquido amniótico puede experimentar una comprensión gástrica que produzca disrupción de la pared con hermanamiento de la mucosa, quedando propenso a la perforación al iniciar la alimentación. Puede también ocurrir dilatación por atrapamiento the aire en casos de plicatura gástrica en posición supina, lo que sumado a una eventual descoordinación entre el cardias y el píloro pueden producir el mismo resultado.

El cuadro clínico es bastante característico y debe sospecharse frente a la aparición súbita de 
distensión abdominal, dificultad respiratoria, vómitos y dificultad para alimentarse en un niño generalmente prematuro y sano hasta ese momento. El diagnóstico se confirma con la radiologia de abdomen simple en posición vertical que muestra neumoperitoneo a tensión, colapso de las vísceras, las que penden junto con el hígado en posición central y nivel líquido en la parte inferior del abdomen. La radiología permite, además, demostrar și hay permeabilidad del tubo digestivo.

El tratamiento es quirúrgico e incluye corrección del desbalance hidroelectrolítico $y$ asistencia respiratoria que se pueda requerir. La cirugía está plenamente indicada una vez demostrado el neumoperitoneo; en el procedimiento debe explorarse prolijamente la cavidad peritoneal buscando perforaciones múltiples y la reparación del defecto debe hacerse mediante la sutura por planos, siendo excepcional que se requiera una resección intestinal. La mortalidad es cercana a $50 \%$ en RN pretérmino y algo menor en los nacidos a término.

En nuestro caso clínico se dieron algunas circunstancias y elementos de los descritos: prematurez, malformación intestinal, alteración del tránsito y cventual dilatación gástrica. La lesión se produjo en la zona más vulnerable, desprovista de músculo y serosa y se repitió por segunda vez debido a la persistencia de la malformación que condicionaba la obstrucción. No tenemos explicación para la perforación del intestino delgado. Tal parece que el problema de las perforaciones fue superado una vez que se permitió un tránsito intestinal expedito. La causa de muerte fue una peritonitis por $E$. Coli, no relacionada directamente con la perforación gastrointestinal.

\section{RESUMEN}

La perforación gástrica es poco frecuente en nuestro medio. A propósito de un caso típico de
Ia maternidad del Hospital Clínico de la Universidad de Chile y resuelto en el Hospital Roberto del Río mediante dos intervenciones quirúrgicas, se analiza la etiopatogenia y tratamiento. El caso clínico correspondió a un prematuro que sufrió neumoperitoneo aguda y a tensión, secundario a una perforación gástrica que se repitió en una segunda oportunidad. En forma cuncomitante presentaba una malrotación intestinal que debe haber jugado un rol fundamental en el desenca. denamiento de la enfermedad. El tratumiento quirúrgico fue exitoso, pero el nirio falleció posteriormente por una peritonitis de causa no precisada en la necropsia.

\section{REFERENCIAS}

1. Izzo $C_{\text {. }}$ Det Rio A.: Perforación gastro-intestinal espontánca del recién nacido. Rev Chil Pediatr $1964 ; 35 \div 418425$.

2. Robarts F.H.: Neonatal perforations of the stomach. Kinder Chirurgie 1968; (Suppl) 5: 62.75.

3. Castleton K.B. Iatch F.F.: Idiopatic perfotation of the stomach іл the ncwborn. Arch Surg 1958. 76: 874-878.

4. Shaw A., Blanc W.A., Santulli T.V., Kaiser G.: Spontaneous rupture of the stomach in the newborn. A clinical and exploratory study. Surgery $1965 ; 58: 561.565$

5. Lloyd J.R.: The etiology of gastrointestinal perfutation in the newborn, J Pediatr Surg 1969; 4: $77-84$.

6. Helardot P.G., El Kaubi Ph., Rapin C.G.: Les perforations gastriques neonatales. Chir Pediatr 1984: 25: $169-175$.

7. Tabet J.L., Felman A.H. De Busk F.L.: Gastrointestinal surgical enjergencies in the newborm infant. J Pediatr 1970; 76: 783-797.

8. Holgrsen $1 . O$.: The etiology of spontaneous gastric perforation of the newbom: a reevaluation, J Pcdiatr Sutg 1981: 16:608-613.

9. Urgencus neonatales quinigicas. Haller.Talbot: Barcelona, Fid. Jims. 1974; pp. 200-205.

10. Givonich A., Doberti A.: Perforaciones gastrointestinales en el recién nacido. Rev Chil Pediatr $1966 ; 37: 160-166$.

11. Léniz M., Steeger A.: Noumoperitoneo espontáneo en un recién nacido. Rev Chil Pediatr 1963; 34: 39.41 . 